\title{
UMA ABORDAGEM HISTÓRICA DE MATRIZES PARA O USO EM SALA DE AULA
}

\section{THE USE OF A HISTORICAL APPROACH ABOUT MATRICES IN THE CLASSROOM}

\author{
Lucas Antonio Mendes de Lima ${ }^{1}$ \\ Universidade do Estado do Pará \\ Mayara Gabriella Grangeiro Pereira ${ }^{2}$ \\ Universidade do Estado do Pará \\ Miguel Chaquiam ${ }^{3}$ \\ Universidade do Estado do Pará
}

\begin{abstract}
Resumo
Motivados por meio das reuniões do Grupo de Pesquisa em História, Educação e Matemática na Amazônia (GHEMAZ), juntamente com as diversas participações em eventos que abordam História da Matemática e desenvolvimento das disciplinas de Pratica de Ensino I e II, nos propomos em elaborar um trabalho que pudesse auxiliar o professor da Educação Básica no ensino de Matemática utilizando a História da Matemática como recurso didático, tendo como base a pesquisa a bibliográfica, de cunho histórico. Ressaltamos que este trabalho é parte do nosso Trabalho de Conclusão de Curso intitulado História da Matemática: contribuições para o ensino de Funções, Equação Quadrática e Matrizes, trabalho cuja constituição foi baseada no diagrama metodológico de Chaquiam (2017), para propor um conjunto de textos envolvendo História da Matemática que possam ser utilizados em sala de aula da Educação Básica durante o processo de ensino de conteúdos matemáticos e, quanto ao recorte, escolhemos o conteúdo de matrizes que foi adaptado para compor este artigo. Sobre o texto evidenciamos Arthur Cayley como personagem principal, seus contemporâneos em ordem cronológica, seus traços biográficos, evolução do conteúdo de matrizes e concepções referentes a esse conteúdo e aos personagens escolhidos. Por fim, destacamos a justificativa que foi apresentada por Cayley, referente a multiplicação matricial e o por que ela se resume em adição de produtos, assim como, a álgebra desenvolvida para as matrizes. Além disso, outro ponto a ser ressaltado é a correlação entre os conteúdos de matrizes, sistemas de equações lineares e determinantes, visto que estes possuem diversas associações. É possível evidenciar no trabalho com um todo a integração entre história e matemática, com a demarcação em tempo e espaço por meio da apresentação fatos históricos ocorridos em torno do período de vida de um dos personagens que contribuíram para a evolução do conteúdo matemático, neste caso, de matrizes.
\end{abstract}

Palavras-chave: Matemática; História da Matemática; Ensino de Matemática; Matrizes

\footnotetext{
${ }^{1}$ lucasamlima@outlook.com

2 maygabriella@ hotmail.com

3 miguelchaquiam@gmail.com
} 


\begin{abstract}
Motivated through the meetings of the Research Group in History, Education and Mathematics in Amazonia (GHEMAZ), together with many participations in events related to the History of Mathematics and the disciplines of Teaching Practice I and II, we aimed to elaborate a work that could help teachers in the teaching of mathematics in Elementary Schools through the use of the History of Mathematics as a didactical resource. We emphasize that this paper is part of one research entitled History of Mathematics: contributions to the teaching of fractions, quadratic equations and matrices, which was based on the methodological diagram of Chaquiam (2017) that proposed a set of historical texts of mathematical development that could be used in elementary schools. About matrices, we considered Arthur Cayley as the main character, his contemporary (organized in chronological order), his biographical traces, the evolution of the content and some conceptions related to this content according to the chosen characters. Then, we show Cayley's justification for matrix multiplication and the reason why he was restricted to addition of products, as well as the algebra developed for the matrices. We also presented the correlation between matrices, linear equations and determinants. It is possible to verify in this work the integration between history and mathematics according to the space and time demarcation of historical facts that happened in the life of one of the characters that truly contributed to the evolution of mathematical knowledge, in this case, matrices.
\end{abstract}

Keywords: Mathematics, History of Mathematics, Teaching of Mathematics, Matrices.

\title{
Introdução
}

As motivações que nos levaram a escrever este trabalho se deram por intermédio dos debates nas reuniões do Grupo de Pesquisa em História, Educação e Matemática na Amazônia (GHEMAZ), vinculada a Universidade do Estado do Pará (UEPA). Assim como, a participação em alguns eventos no decorrer da graduação, entre eles o II Seminário Cearense, onde tivemos o contato com diversos trabalhos que abordam a História da Matemática e suas relações com o Ensino, e a vivencia em sala de aula por meio das disciplinas de Pratica de Ensino I e II.

Dessa forma, tendo em vista como se caracteriza o cenário educacional atualmente e visando propor novas estratégias para melhorar este cenário, buscamos elaborar um trabalho que auxiliasse o professor da Educação Básica no ensino de Matemática utilizando a História.

Com isso, recorremos à pesquisa bibliográfica de cunho histórico, que segundo Gil (2008), é desenvolvido com material elaborado, constituído principalmente de livros e artigos científicos. Objetivando apresentar uma constituição histórica a respeito da evolução do conteúdo de Matrizes para uso em sala de aula na Educação Básica. 
Ressaltamos que este artigo é parte do Trabalho de Conclusão de Curso (TCC), intitulado: A História da Matemática: contribuições para o ensino de Funções, Equação Quadrática e Matrizes, no qual nos baseamos no diagrama metodológico de Chaquiam (2016), para propor um conjunto de textos envolvendo História da Matemática que possam ser utilizados em sala de aula da Educação Básica durante o processo de ensino de conteúdos matemáticos. Sendo assim, escolhemos o conteúdo de Matrizes para ser abordado neste artigo e tomamos por base o diagrama abaixo, Figura 1.

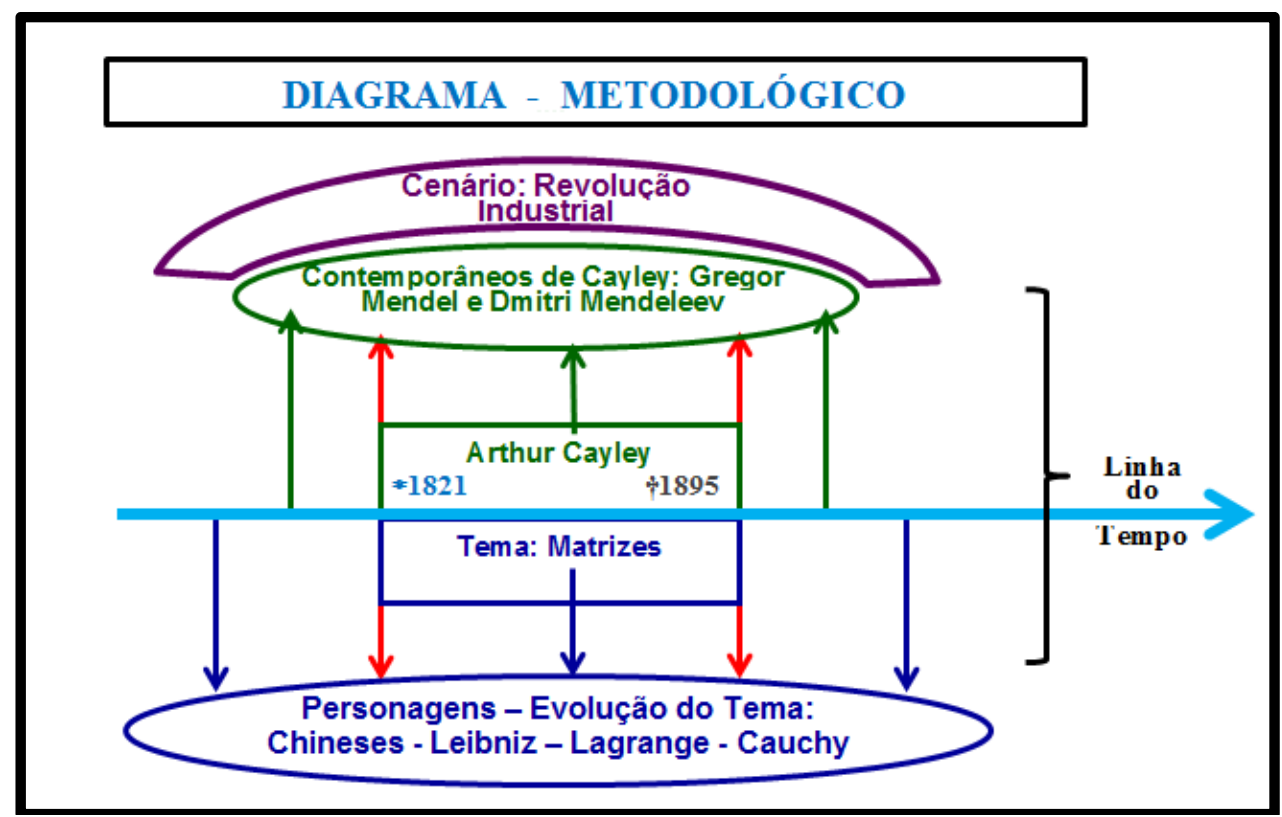

Figura 1 - Diagrama Metodológico: Matrizes

Fonte: Elaborado pelos autores, adaptado de Chaquiam (2017)

Sobre a evolução do conteúdo de Matrizes, optamos por este assunto devido sua importância e utilização no $2^{\circ}$ ano do Ensino Médio e na graduação de Licenciatura em Matemática, onde percebemos por meio da dissertação de Santos (2007) os benefícios que os alunos do Ensino Médio poderiam ter se matrizes e determinantes fossem ensinados levando em consideração o curso histórico dos acontecimentos, descobertas e conceitualizações de cada conteúdo.

O texto apresenta-se dividido na seguinte ordem: contexto histórico do personagem principal, Arthur Cayley, seus contemporâneos em ordem cronológica, seus traços biográficos, evolução do conteúdo de matrizes e por fim outros olhares referentes a esse conteúdo. 


\section{Cenário Mundial - Revolução Industrial}

A euforia tecnológica propagada pela Revolução Industrial marcou o século XIX como um período de ascensão de diversas áreas do conhecimento. Os assuntos de ordem científica e estética passaram a despertar o interesse de um grande público. Várias nações criaram instituições que buscavam o desenvolvimento de estudos em prol do progresso da ciência.

As ciências exatas ganharam grande impulso na medida em que o desenvolvimento tecnológico vinculava-se com o desenvolvimento industrial. A Física, a Química fina e a Metalurgia foram as principais áreas de desenvolvimento científico. Influenciadas por essas mudanças, as ciências humanas também observaram o surgimento de novas áreas como a Sociologia e a Psicologia.

$\mathrm{Na}$ esfera artística, o individualismo e o ritmo frenético dos ambientes urbanos impulsionaram a criação de novos movimentos, como o: Romantismo que criticava as mudanças da sociedade industrial e buscava o refúgio na vida próxima à natureza e a exaltação dos sentimentos amorosos; o Parnasianismo que pautava uma concepção de elogio ao belo, considerando que a arte seria um campo autônomo que não deveria se ocupar dos conflitos e horrores da condição humana; e o Naturalismo que valorizava as contradições das relações humanas e a reflexão do mundo vivido. Nesse mesmo período o pensamento marxista se estabelecia, sendo um forte influenciador nas literaturas.

\section{Contemporâneos de Arthur Cayley}

Uma vez que vimos como se caracterizava o cenário mundial da época em que viveu Cayley, destacamos a seguir alguns personagens que foram seus contemporâneos e que contribuíram para o meio cientifico: Gregor Mendel (1822-1884), Dmitri Mendeleev (1834-1907), Henri Poincarè (1854-1912) e Heinrich Rudolf Hertz (18571894). É importante frisar que nos baseamos nos livros de Eves (1997) e no Dicionário de biografias científicas (2007) para fazer um relato breve sobre cada personagem escolhido.

Iniciando com Gregor Mendel, que nasceu em 20 de julho de 1822, num pequeno povoado chamado Heinzendorf, na atual Áustria. Tinha como nome de batismo Johann Mendel, mas após ingressar para a ordem religiosa dos agostinianos mudou o nome para Gregor Mendel. 
Entre os anos de 1851 e 1853 estudou História Natural na Universidade de Viena, adquirindo muitos conhecimentos que seriam de extrema importância para descobertas que mudariam o rumo da Biologia e posteriormente serviriam de base para o desenvolvimento de suas teorias (leis da Genética).

Suas pesquisas conduziram ao descobrimento das primeiras leis quantitativas da Biologia, contribuindo para a evolução das ciências, sua teoria principal era a de que as características das plantas deviam-se a elementos hereditários (atualmente conhecidos como genes).

Dando continuidade aos contemporâneos, temos Dmitri Mendeleev que nasceu na cidade de Tobolsk na Sibéria no dia 8 de fevereiro de 1834, falecendo no dia 2 de fevereiro de 1907 em São Petersburgo. Na escola, se destacou na área das Ciências desde cedo. Ao ingressar o ensino superior interessou-se pela química e concluiu sua graduação como sendo o melhor de seu curso.

Enquanto escrevia seu livro de química inorgânica, Mendeleev organizou os elementos conhecidos, criando uma carta para cada um, contendo em cada uma o símbolo do elemento, a massa atómica e as suas propriedades químicas e físicas. Organizou-as em ordem crescente de massas atómicas, agrupando-as em elementos de propriedades semelhantes.

A tabela de Mendeleev serviu de base para a elaboração da tabela periódica atual, que além de catalogar os 118 elementos conhecidos, fornece inúmeras informações sobre o comportamento de cada um.

Segundo Eves (1997) Henri Poincaré liderou e enriqueceu uma gama espantosa de assuntos na matemática, sendo um escritor numeroso, deixou mais de 30 livros e 500 artigos técnicos. Sua tese de doutorado sobre equações diferenciais dizia a respeito a teoremas de existência, que levou ao desenvolvimento da teoria das funções automórficas e, em particular, das funções zeta-fuchsianas que podem ser usadas para resolver equações diferenciais lineares de segunda ordem com coeficientes algébricos.

Poincaré nunca se preocupou em permanecer por muito tempo em um campo só tendo interesse e contribuições, por exemplo, na topologia combinatória, geometria não euclidiana, óptica, eletricidade, telegrafia, capilaridade, elasticidade, termodinâmica, teoria do potencial, teoria quântica, teoria da relatividade e cosmogonia. 
E por fim, o último contemporâneo eleito, Heinrich Rudolf Hertz que foi um físico alemão, nascido no dia 22 de Fevereiro de 1857 e falecendo no dia 1 de Janeiro de 1894. Foi o responsável pela descoberta das ondas eletromagnéticas em 1888, em sua homenagem foi atribuído ao seu nome à unidade de frequência "Hertz".

A respeito das propriedades das ondas eletromagnéticas Hertz, descobriu que a sua velocidade de propagação é igual à velocidade da luz no vácuo, que têm comportamento semelhante ao da luz, e que oscilam num plano que contém a direção de propagação. Demonstrou a refração, a reflexão e a polarização das ondas. Provou, ainda, que os materiais condutores elétricos refletem as ondas e que os nãos condutores favorecem a passagem delas. $\mathrm{O}$ seu trabalho permitiu o desenvolvimento do rádio, da televisão e do radar.

\section{Traços Biográficos de Cayley}

Arthur Cayley foi um matemático britânico. De acordo Eves (1997), 16 de agosto de 1821, na cidade de Richmond, Surrey. No início de seus estudos, teve passagem pelo Trinity College. Por volta de vinte anos de idade o matemático graduouse como primeiro wrangler nos tripos de matemática. Além das atividades na área de matemática, Arthur também exerceu a advocacia durante no intervalo de tempo de sete anos, período em que publicou entre 200 e 300 artigos durante sua prática jurídica. Além disso, também possuía habilidades em Inglês, Grego, Frances, Alemão e Italiano.

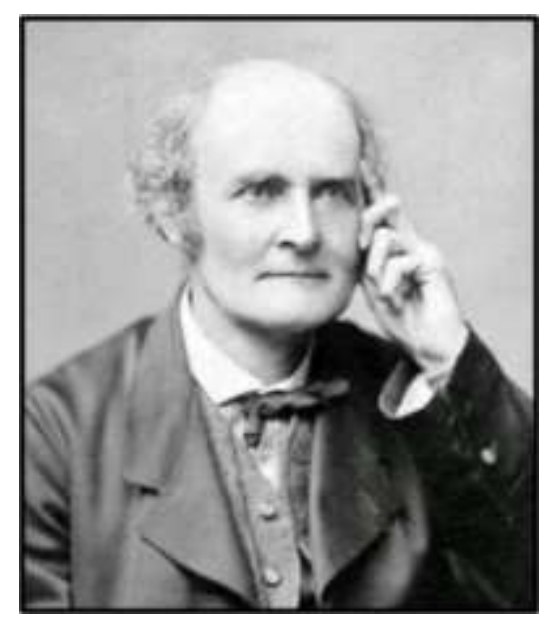

Figura 2 - Arthur Cayley 
Suas principais contribuições, segundo Eves (1997), foram em diversos campos da Matemática. Entre eles, destacam-se a geometria analítica, a teoria das transformações, teoria dos determinantes, geometria de dimensão superiores, teoria da partição, teoria das curvas e superfícies, o estudo de formas binarias e ternarias e teoria das funções abelianas, tetas e elípticas e as matrizes.

Evidencia-se entre suas publicações o livro, "Menoir on the Theory of Matrices" no ano de 1858. Este livro revelava o poder da álgebra matricial a partir de algumas considerações acerca das matrizes de Cayley. Por isso e por suas contribuições Cayley foi chamado "o matemático dos matemáticos". Assim, após seus feitos, ele faleceu no dia 26 de janeiro de 1895, em Cambridge, Cambridgeshire, Inglaterra.

\section{Evolução do conteúdo de Matrizes}

Os Chineses, eram povos das civilizações antigas, que segundo Boyer (1996), surgiram 1000 a.C. No início de seus estudos desenvolveram livros relacionados ao tratamento de cálculos astronômicos, assim como, promoveram contribuições a respeito das propriedades relacionadas ao triangulo retângulo, frações, geometria relacionada à álgebra e a na aritmética.

Conteúdos estes, abordados em grande parte pelo livro chinês mais influente de matemática denominado de "Chui-Chang Suan-Shu" ou "Nove capítulos sobre a arte matemática". Nele os Chineses apresentam os primeiros registros de um quadrado mágico, disposto da seguinte forma:

$$
\begin{array}{|lll|}
4 & 9 & 2 \\
3 & 5 & 7 \\
8 & 1 & 6 \\
\hline
\end{array}
$$

O qual motivou aquele povo a uma relação deste diagrama à resolução de sistemas de equações lineares simultâneas. Com segue o raciocínio a baixo:

$$
\left\{\begin{array}{l}
3 x+2 y+z=39 \\
2 x+3 y+z=34 \\
x+2 y+3 z=26
\end{array}\right.
$$

Para isso, eram efetuadas operações sobre colunas na matriz 


\begin{tabular}{|ccc|}
\hline 1 & 2 & 3 \\
2 & 3 & 2 \\
3 & 1 & 1 \\
26 & 34 & 39
\end{tabular}$\quad$ para reduzi-la a $\quad$\begin{tabular}{ccc|}
0 & 0 & 3 \\
0 & 5 & 2 \\
36 & 1 & 1 \\
99 & 24 & 39 \\
\hline
\end{tabular}

A fim de representar as equações na forma $36 z=99 ; 5 y+z=24$ e $3 x+2 y+z=$ 39. Das quais calcula-se os respectivos valores de $\mathrm{z}$, y e $\mathrm{x}$.

Nesse sentido verifica-se uma caracterização inicial de matrizes, de forma intuitiva, associada ao diagrama do quadrado mágico. Como consequência objetivavam solucionar o sistema de equações lineares simultâneas por meio do escalonamento para se chegar a matriz escalonada.

Gottfried Wilhelm Leibniz (1646 - 1716), de acordo com Eves (1997), nasceu em 1646, na cidade de Leipzig. Bastante criança desenvolveu habilidades em grego e latim, assim como, com o tempo passou a dominar todo o conhecimento referente a corrente matemática, filosófica, teológica e ramos do direito.

No que refere-se ao conteúdo de matrizes, segundo Boyer (1996), Leibniz faz uma associação ao método de determinante. Assim, no ano de 1693, apresenta por meio de cartas a L'Hospital uma caracterização para indicar linhas e colunas, originadas a partir de equações lineares simultâneas. Como segue:

$$
\left\{\begin{array} { l } 
{ 1 0 + 1 1 x + 1 2 y = 0 } \\
{ 2 0 + 2 1 x + 2 2 y = 0 } \\
{ 3 0 + 3 1 x + 3 2 y = 0 }
\end{array} \text { ou } \quad \left\{\begin{array}{l}
1_{0}+1_{1}+1_{2}=0 \\
2_{0}+2_{1}+2_{2}=0 \\
3_{0}+3_{1}+3_{2}=0
\end{array}\right.\right.
$$

Escreviam isso como:

$$
\left\{\begin{array}{l}
a_{1}+b_{1} x+c_{1} y=0 \\
a_{2}+a_{2} x+c_{2} y=0 \\
a_{3}+b_{3} x+c_{3} y=0
\end{array}\right.
$$

Se as equações fossem consistentes então

$$
\begin{aligned}
& 1_{0} \cdot 2{ }_{1} \cdot 3_{2}+1_{0} \cdot 2 \cdot 3_{2} \cdot 3_{1} \\
& 1_{1} \cdot 2{ }_{2} \cdot 3_{0}=1_{1} \cdot 2_{0} \cdot 3_{2}, \\
& 1_{2} \cdot 2_{0} \cdot 3_{1}+1_{2} \cdot 2 \cdot 3_{0}
\end{aligned}
$$

Noção esta que equivale ao enunciado atual referente ao determinante, escrito por 


$$
\left|\begin{array}{lll}
a_{1} & b_{1} & c_{1} \\
a_{2} & b_{2} & c_{2} \\
a_{3} & b_{3} & c_{3}
\end{array}\right|=0
$$

Com isso, nota-se que assim como no estudo dos chineses, por Leibniz as matrizes são evidenciadas a partir de equações lineares simultâneas. Porém, em suas contribuições este autor enfatiza a indicação para linhas e colunas de matrizes afim de compor o método do determinante, o qual foi apresentado com notações próximas as quais utilizamos atualmente.

Josep Louis Lagrange, segundo Eves (1997), nasceu no ano de 1736, em Turim, Itália. Este é considerado ao lado de Euler o maior matemático do século XVIII, isto, por conta de críticas à Análise, por meio de sua grande obra Théorie des Functions Analytiques Contenant les Principes du Calcul Différentiel, além das atribuições à série de Taylor.

De acordo com Boyer (1996), outro trabalho desenvolvido por ele que merece destaque é o artigo intitulado "Solutions Analytiques de qualques problèms sur les pyramides triangulares", publicado em 1975. O qual apresentou resultados analíticos para o cálculo de área de um triangulo e o volume de um tetraedro, denotados respectivamente por $\mathrm{A} \mathrm{e} \mathrm{V}$, como temos a seguir:

$$
A=\left(\frac{1}{2}\right)\left[\begin{array}{lll}
x 1 & y 1 & 1 \\
x 2 & y 2 & 1 \\
x 3 & y 3 & 1
\end{array}\right] \quad \text { e } \quad V=\left(\frac{1}{6}\right)\left[\begin{array}{ccccc}
x 1 & y 1 & z 1 & \cdots & 1 \\
x 2 & y 2 & z 2 & \ddots & 1 \\
x 4 & y 4 & z 4 & \cdots & 1
\end{array}\right]
$$

A partir disso, ele concluiu que os elementos que compõe as matrizes (denominação atual), representavam os vértices do triangulo e do tetraedro e caracterizam coordenadas de pontos no espaço, como verifica-se na fórmula para o cálculo de A e V.

Assim, verifica-se que Lagrange buscou artifícios analíticos para caracterizar os elementos que compõe as linhas e colunas das matrizes, afim de justificar que dependendo do campo matemático abordado não representam apenas "números tabulados".

Algustin Louiz Cauchy, de acordo com Eves (1997), nasceu no ano de 1789, em Paris. Suas obras abrangem além de vários livros uma gama de 789 artigos científicos, dos quais, destacou as produções em Séries Infinitas, Teoria das Funções Reais Complexas, Equações Diferenciais, Probabilidade, Física-Matemática e Determinantes. 
Dentre estes estudos, foi o matemático que mais desenvolveu estudos acerca dos Determinantes, consequentemente apresentou considerações significativas a respeito das matrizes. Entre as contribuições, a demonstração de um teorema que envolvem ambos os conteúdos. O qual foi proposto da seguinte maneira:

"Se A e B são matrizes $n \times n$, então $|A . B|=|A| .|B| "$

Assim, a partir do teorema exposto, verifica-se que apesar de ele não citar a nomenclatura "ordem", mas considera que A e B são matrizes $n \times n$ (classificação intuitiva das matrizes quadradas), caracterizando mais um elemento para construção deste estudo. Além disso, destaca-se a relação entre o determinante e as matrizes, a qual, também foi citada na abordagem feita por Leibniz.

Arthur Cayley, segundo Boyer (1996), em sua grande obra "Menoir on the Theory of Matrices" a respeito da teoria das matrizes, do ano de 1858, revela o poder da álgebra matricial. A qual surge a partir da Teoria das Transformações Lineares Simultâneas, representada a seguir de maneira análoga:

Inicialmente considere as transformações $T_{1}$ e $T_{2}$

$$
T_{1}=\left\{\begin{array}{l}
\mathrm{x}^{\prime}=\mathrm{ax}+\mathrm{by} \\
\mathrm{y}^{\prime}=\mathrm{cx}+\mathrm{dy}
\end{array} \text { e } T_{2}=\left\{\begin{array}{l}
\mathrm{x}^{\prime \prime}=\mathrm{Ax}^{\prime}+\mathrm{By}^{\prime} \\
\mathrm{y}^{\prime \prime}=\mathrm{Cx}^{\prime}+\mathrm{Dy}^{\prime}
\end{array}\right.\right.
$$

Verifique que a segunda transformação apresenta incógnitas presentes na primeira. Assim, se aplicarmos $T_{1}$ em $T_{2}$, temos:

$$
T_{1} T_{2}=\left\{\begin{array}{l}
\mathrm{x}^{\prime \prime}=(\mathrm{Aa}+\mathrm{Bc}) \mathrm{x}+(\mathrm{Ab}+\mathrm{Bd}) \mathrm{y} \\
\mathrm{y}^{\prime \prime}=(\mathrm{Ca}+\mathrm{Dc}) \mathrm{x}+(\mathrm{Cb}+\mathrm{Db}) \mathrm{y}
\end{array}\right.
$$

O resultado expresso na linguagem da matriz recai na multiplicação entre matrizes

$$
\left(x^{\prime \prime}, y^{\prime \prime}\right)=\left(\begin{array}{ll}
A a+B c & A b+B d \\
C a+D c & C b+D d
\end{array}\right) \cdot\left(\begin{array}{l}
x \\
y
\end{array}\right)
$$

Ou ainda, da seguinte forma:

$$
\left(x^{\prime \prime}, y^{\prime \prime}\right)=\left(\begin{array}{ll}
A & B \\
C & D
\end{array}\right) \cdot\left(\begin{array}{ll}
a & b \\
c & d
\end{array}\right) \cdot\left(\begin{array}{l}
x \\
y
\end{array}\right)
$$

A partir desta construção, Cayley mostrou que ao inverter a ordem das matrizes com letras maiúsculas pelas minúsculas o produto era diferente. Ou seja, não conseguiase retornar ao sistema de origem. Com isso, mostrou que a multiplicação matricial não é comutativa. 
Além da multiplicação, ele também apresentou uma álgebra para adição de matrizes (de iguais dimensões). A qual, é obtida somando-se os elementos correspondentes das matrizes, como segue:

$$
\left(\begin{array}{ll}
A & B \\
C & D
\end{array}\right)+\left(\begin{array}{ll}
a & b \\
c & d
\end{array}\right)=\left(\begin{array}{ll}
A+a & B+b \\
C+c & D+d
\end{array}\right)
$$

A multiplicação de uma matriz por um escalar k é definida pela relação a baixo:

$$
k \cdot\left(\begin{array}{ll}
\mathrm{a} & \mathrm{b} \\
\mathrm{c} & \mathrm{d}
\end{array}\right)=\left(\begin{array}{ll}
\mathrm{ka} & \mathrm{kb} \\
\mathrm{kc} & \mathrm{kd}
\end{array}\right) \cdot\left(\begin{array}{l}
\mathrm{x} \\
\mathrm{y}
\end{array}\right)
$$

Por fim, expos a definição de matriz identidade, denotada por I, a qual pode ser associada ao "elemento neutro" da multiplicação matricial. Assim como, a matriz quadrada nula, como o "elemento neutro" da adição de matrizes. As quais, denotam-se respectivamente por:

$$
\left(\begin{array}{ll}
1 & 0 \\
0 & 1
\end{array}\right) \text { e }\left(\begin{array}{ll}
0 & 0 \\
0 & 0
\end{array}\right)
$$

Com isso, nota-se o quão influente foi Arthur Euler para o estudo das Matrizes. Influência esta notada com as suas contribuições a respeito da definição de multiplicação matricial a partir das transformações lineares, assim como, para adição de matrizes (de iguais dimensões), multiplicação por escalar, além das noções para matriz identidade e matriz nula.

\section{Outros olhares sobre o tema}

Abordamos a seguir uma dissertação e uma citação referente aos respectivos pontos: a primeira com ênfase para abordagem histórica das matrizes e outra refere-se a um comentário acerca do Arthur Cayley.

Assim, evidenciamos a dissertação de Santos (2007), na qual, o autor enfatiza a importância da abordagem histórica do conteúdo de matrizes para educação básica.

Além disso, destacamos também o comentário registrado de Charles Hermite, presente no livro do Boyer (1996), após a morte de Cayley: “o talento matemático de Cayley se caracterizou pela careza e extrema elegância da forma analítica; reforçado por uma capacidade incomparável de trabalho ...".

Nesse sentido, apresentamos a seguir algumas ponderações a respeito do texto desenvolvido. 


\section{Considerações}

Ao compreendermos a construção apresentada anteriormente a respeito de Matrizes, entendemos que a História da Matemática não deve ser interpretada como separada do ensino da matemática, mas, como um recurso complementar para abordagem dos conteúdos, associado a resolução de problemas e as demais tendências em Educação Matemática.

Neste sentido, a respeito da evolução dos estudos referentes as Matrizes destacamos a noção intuitiva desenvolvida pelos Chineses relacionada ao quadrado mágico. Associada a indicação dos elementos das linhas e colunas para compor o método do determinante de Leibniz.

Seguido da interpretação geométrica, exposta por Lagrange ao caracterizar os elementos das matrizes como coordenadas de pontos no espaço. Além disso, destaca-se a noção de Cauchy a respeito da ordem das matrizes e sua relação com o determinante por meio do teorema exposto.

Por fim, evidenciou-se Cayley, como o matemático que apresentou a álgebra para o estudo das matrizes, além do fato, de considerar que a abordagem de matrizes deve preceder determinante.

Observou-se que a proposta apresentada por Chaquiam (2017), elaborar um teto a partir da construção de um diagrama metodológico orientador, é viável e o referido diagrama nos proporciona uma visão unitária do processo. Além disso, as pesquisas nos indicam a existência de diversos caminhos e possibilidades de construção de uma história a respeito de uma temática. Por outro lado, foi possível observar que ainda temos poucos textos voltados para o uso da história da matemática como recurso didático em sala de aula.

\section{Referências}

BOYER, C. História da Matemática. São Paulo: Blucher, 1996.

CHAQUIAM, M. Um diagrama, um texto. In: MENDES, I. A. \& CHAQUIAM, M. História nas aulas de Matemática: fundamentos e sugestões didáticas para professores. Belém: SBHMat, 2016.

CHAQUIAM, M. Ensaios Temáticos: História e matemática em sala de aula. Belém: SBEM/PA, 2017. 
D’AMBROSIO, U. Por que e Como Ensinar História da Matemática. In: FOSSA, J. A. (Org) Facetas do Diamante. Rio Claro - SP: Ed. SBHMat, 2000.

D’AMBROSIO, U. A Interface entre História e Matemática: Uma Visão HistóricoPedagógica. REMATEC. Natal (RN): EDUFRN, 2013.

EVES, H. Introdução a História da Matemática. Campinas (SP): Editora da UNICAMP, 1997

GIL, A. C. Como elaborar projeto de pesquisa. São Paulo: Editora Atlas S.A. 2008.

GILLISPIE, C. C. Dicionário de biografias cientificas. Tradução Carlos Almeida Pereira... [et al.]. - Rio de Janeiro: Contraponto, 2007.

SANTOS, R. N. Uma breve história do desenvolvimento das teorias dos determinantes e das matrizes. 2007. 42 f. Dissertação - Instituto de Matemática e Estatística da Universidade de São Paulo. São Paulo, 2007. 